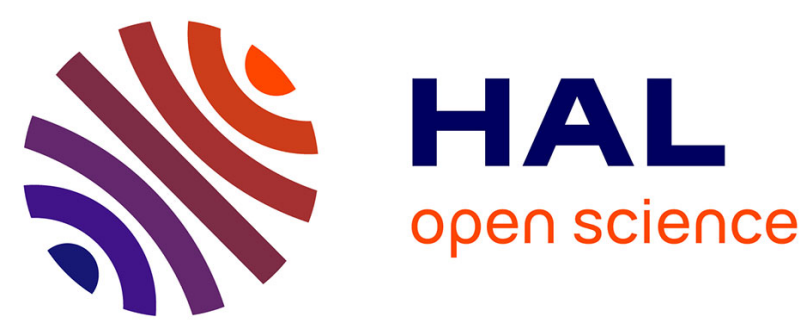

\title{
Discrete Power Semiconductor Losses Versus Junction Temperature Estimation Based on Thermal Impedance Curves
}

Do Phuong Uyen Tran, Stéphane Lefebvre, Yvan Avenas

\section{- To cite this version:}

Do Phuong Uyen Tran, Stéphane Lefebvre, Yvan Avenas. Discrete Power Semiconductor Losses Versus Junction Temperature Estimation Based on Thermal Impedance Curves. IEEE Transactions on Components, Packaging and Manufacturing Technology, 2020, 10 (1), pp.79-87. 10.1109/TCPMT.2019.2939617 . hal-03260263

\section{HAL Id: hal-03260263 https://hal.science/hal-03260263}

Submitted on 14 Oct 2021

HAL is a multi-disciplinary open access archive for the deposit and dissemination of scientific research documents, whether they are published or not. The documents may come from teaching and research institutions in France or abroad, or from public or private research centers.
L'archive ouverte pluridisciplinaire HAL, est destinée au dépôt et à la diffusion de documents scientifiques de niveau recherche, publiés ou non, émanant des établissements d'enseignement et de recherche français ou étrangers, des laboratoires publics ou privés. 


\title{
Discrete power semi-conductor losses vs junction temperature estimation based on thermal impedance curves
}

\author{
Do Phuong Uyen TRAN ${ }^{1}$, Stéphane LEFEBVRE ${ }^{2}$, Yvan AVENAS ${ }^{1}$ \\ ${ }^{1}$ Univ. Grenoble Alpes, CNRS, Grenoble INP, G2Elab, F-38000 Grenoble, France \\ ${ }^{2}$ SATIE, ENS Paris Saclay, CNAM, CNRS F-94230 Cachan, France
}

\begin{abstract}
Loss estimation in power semi-conductor components is an important research topic for a long time with various methods that have been proposed in the literature. Although these methods have been implemented and allow estimating the losses with satisfactory results, the junction temperature of the component is generally controlled poorly while it influences the losses. This paper presents a dynamic calorimetric method used to estimate the losses in power semi-conductor devices, taking into account their evolution as a function of the junction temperature. The proposed method is based on the temperature measurement in a metal block fixed under the component. Through a thermal model based on the notion of thermal impedance, the transient temperature measurement in the block allows determining the losses as well as the junction temperature of the device. In order to size the test bench, determine the robustness of this method with respect to measurement or implementation uncertainties (heat transfer coefficients, thermal conductivity of materials), thermal simulations were performed using Flotherm software. Due to the presence of noise in the measurements, post signal processing is also necessary. By applying the chosen setting, a corresponding experiment was conducted to verify the proposed method. This experiment showed promising results for both loss and junction temperature determinations.
\end{abstract}

Index Terms-Calorimetry, junction temperature, losses, power semi-conductor, thermal impedance.

\section{INTRODUCTION}

$\mathrm{T}$ HE estimation of the losses in power semi-conductor components is an essential data for estimating the efficiency of static converters and for choosing the thermal management system to associate with the converter.

Usually, the switching losses can be estimated by measuring current and voltage waveforms with the double-pulse method [1], [2]. However, because of additional parasitic elements related to the insertion of sensors in the switching cell, and the high switching speeds of wide band gap semi-conductors, the electrical measurements become extremely complex to implement. Huge errors can be generated if the current / voltage measurements are not carried out perfectly (large bandwidth, and delay compensation between current and voltage probes).

In order to get rid of electrical measurements during switching and to avoid the bandwidth requirements and/or the temporal synchronization between current and voltage measurements, several studies have focused on electrical measurements of total losses at the converter level [3] - [5], or on calorimetric measurements [6] - [14].

On the one hand, the traditional loss measurement method is to measure the input and output powers of the studied converter. However, this method requires a power analyser with very high precision and large bandwidth, and can cause significant errors for high-efficiency converters. To avoid these difficulties, the opposition method has been used to estimate the losses by measuring only the input power on a DC bus [3]. The opposition method allows also minimizing the power supply that provides only the total losses in the system and estimating these total losses for a given operating point. Moreover, the conduction, turn-on and turn-off losses can be separated by using hard switching and Zero Voltage Switching (ZVS) at different frequencies [4], [5]. The losses in semi-conductor devices can be estimated by subtracting the other losses, such as losses in passive components or in electrical connections. Unfortunately, these losses are sometimes difficult to measure and/or estimate.

On the other hand, the heat flux dissipated by the devices can be estimated to determine the losses. Calorimetric methods consist of measuring several temperatures and, through a thermal model, estimating the thermal flux dissipated by the components.

Conventional calorimetry is based on the estimation of the heat flux generated by the component in steady state in order to deduce the total dissipated losses. Several studies have been already done such as measurement of the temperature increase of a fluid (air, water) in a calorimetric chamber [6], [7], temperature measurement in a metal bar on which the component is placed [8], local temperature measurement using IR camera [9] or direct measurement by a heat flux sensor[10]. However, since the measurements are carried out under stationary conditions, the duration of the measurements may be excessive.

Another method, the dynamic calorimetry, is based on the transient measurement of the temperature in a thermally conductive block. Therefore, the measurement time is shorter in comparison with conventional steady-state calorimetry. This method has been used for devices with high switching speed when insertion of current probes was prohibited, for example for GaN HEMTs [11], for SiC BJT [12] or for SiC MOSFET and diodes used in soft switching [13]. Moreover, the 
conduction, turn-on and turn-off losses can be also estimated separately by adding a switch in series with the top leg of a halfbridge circuit and modifying the modulation strategy [13], [14].

Such techniques have been implemented and allow estimating the losses with satisfactory results. Unfortunately, while the double-pulse method allows knowing the working temperature of the device, the opposition method and some calorimetric methods induce an increase of the junction temperature during the measurement and do not allow evaluating the junction temperature at which the losses are estimated. Under these conditions, these measurement methods do not allow directly estimating the losses for a given junction temperature without the use of a specific thermal model.

This article aims to propose and evaluate a method based on dynamic calorimetry to measure the losses in the components and especially to present their evolution according to the junction temperature. In order to evaluate the potentialities of this method, the experimental tests will be carried out, in a first step, considering only the conduction losses. Indeed, in these particular conditions, without having to consider the switching of transistors and / or diodes, the experimental validation is simplified because it frees itself from the difficulties of measurements related to a very constraining electromagnetic environment. The validation of the proposed method in a switching environment will be studied in a later work.

The principle of the proposed method is described in section II. Section III and IV are dedicated to an evaluation of this method through thermal simulations. Eventually, experimental measurements and validations are proposed in the last part.

\section{PRINCIPLE OF The Proposed Method}

Basically, the proposed method consists of fixing the device under test to a metal block and measuring a local temperature in the block to estimate the device losses and junction temperature versus time in operation. It is therefore mandatory to first identify a thermal model of the assembly consisting of the metal block and the component to be characterized and then to use this model with an inverse method to simultaneously estimate the losses and the junction temperature.

\section{A. Thermal model}

To illustrate the proposed transient calorimetric method, a simulation model is implemented using Flotherm software (Fig. 1) with a semi-conductor device mounted on a copper block. According to the datasheet of the chosen MOSFET (IRF640N), the case-to-sink thermal resistance Rthc-s is $0.5 \mathrm{~K} \cdot \mathrm{W}^{-1}$ (with heatsink compound), and the effective contact area $\mathrm{A}$ is 160 $\mathrm{mm}^{2}$. Therefore, the thermal contact resistance between the component and the block is fixed to $10^{-4} \mathrm{~K} \cdot \mathrm{m}^{2} \cdot \mathrm{W}^{-1}$. All the outer faces of the system are in a first step supposed to be thermally insulated. The temperature $T_{l}$ in the metal block is measured beneath the chip. The distance between the device and the temperature sensor is $1 \mathrm{~mm}$. $T_{j}$ is the junction temperature.

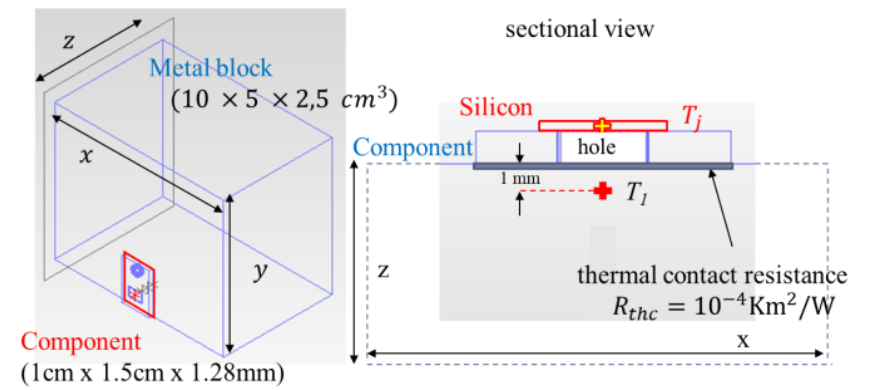

Fig. 1. Geometry of the simulated system with Flotherm software

This method is based on the notion of thermal impedance, which links the temperatures within the assembly to the losses. In the case of a step of dissipated power $P$, the thermal impedance $Z t h_{l}$ from the location of the $T_{l}$ measurement to the ambient, and the thermal impedance $Z t h_{j}$ from the component junction to the ambient are defined respectively by:

$$
\begin{aligned}
& Z t h_{1}(t)=\frac{T_{1}(t)-T_{1}(0)}{P} \\
& Z t h_{j}(t)=\frac{T_{j}(t)-T_{j}(0)}{P}
\end{aligned}
$$

It is necessary to ensure that at the first moment, the system is in thermal equilibrium, which means that $T_{I}(0)=T_{j}(0)$, and these values are considered as the ambient temperature.

In summary, the thermal model is identified by injecting a known power to the system, measuring the temperature within the block and the junction temperature of the component. However, due to the difficulty of directly measuring the junction temperature in practice, an indirect measurement technique using a thermo-sensitive electrical parameter (TSEP) is applied [15]. This measurement will be explained in detail in section V-C.

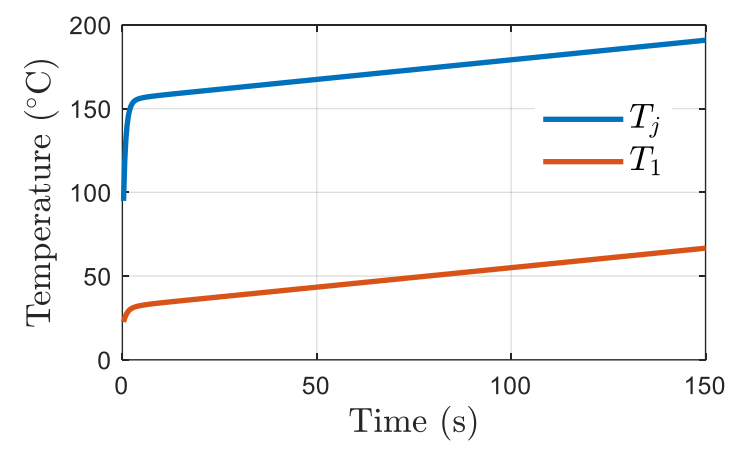

Fig. 2. Temperature evolution as a function of time for $P=100 \mathrm{~W}$

Fig. 2 shows both temperature curves in the time domain for a $100 \mathrm{~W}$ power step. For short times (between 0 and $5 \mathrm{~s}$ ), due to the thermal contact resistance, the junction temperature of the component increases very rapidly. Otherwise, over long times, the temperature curves increase progressively with similar slopes related to the dissipated power. 


\section{B. Estimation of $P\left(T_{j}\right)$}

In operation, the losses $P$ of a power semi-conductor device vary with time. In these conditions, equation (3) relates the power $P$ to the temperature $T\left(T_{l}\right.$ or $\left.T_{j}\right)[16]$ :

$$
\int_{0}^{t} Z \dot{t h}(\tau) \cdot P(t-\tau) \cdot d \tau=T(t)-T(0)=\theta(t)
$$

where $\operatorname{Zth}(\tau)$ is the derivative of the thermal impedance $\operatorname{Zth}(\tau)$.

With the time step $\delta \tau \rightarrow 0$, the convolution integral can be numerically calculated with a Riemann sum as follows:

$$
\begin{gathered}
\sum_{\tau=0}^{\mathrm{t}} Z \operatorname{th}(\tau) \cdot P(t-\tau) \cdot \delta \tau=\theta(t) \\
\underbrace{\left[\begin{array}{cccc}
Z \operatorname{tin}(0) & 0 & \ldots & 0 \\
Z \operatorname{th}(1) & Z \dot{t h}(0) & \ldots & 0 \\
\ldots & \ldots & \ldots & 0 \\
Z \operatorname{th}(N) & Z \dot{t h h}(N-1) \ldots Z \operatorname{th}(0)
\end{array}\right]}_{\text {Zthd }} \cdot \underbrace{\left[\begin{array}{c}
\theta(0) \\
P(1) \\
\ldots \\
P(N)
\end{array}\right]}_{\boldsymbol{P}} \cdot \delta \tau \\
=\left[\begin{array}{c}
\theta(1) \\
\ldots \\
\theta(N)
\end{array}\right]
\end{gathered}
$$

Zthd is the triangular matrix created based on the derivative of the thermal impedance (5). The matrix $\boldsymbol{Z} \boldsymbol{t h} \boldsymbol{d}_{j}$ and $\boldsymbol{Z} \boldsymbol{t h} \boldsymbol{h} \boldsymbol{d}_{\boldsymbol{I}}$ are calculated respectively using thermal impedance $Z t h_{j}$ and $Z t h_{l}$ measured with a power step. Applying for the case of junction temperature increase $\theta_{j}$ and temperature increase within the block $\theta_{1}$, equation (5) can be rewritten as below:

$$
\begin{aligned}
& \theta_{j}=\boldsymbol{Z t h d}_{\mathbf{j}} \cdot P . \delta \tau \\
& \theta_{1}=\boldsymbol{Z t h d}_{\mathbf{1}} \cdot P . \delta \tau
\end{aligned}
$$

Thus, when the power semi-conductor device is in normal operation, the losses can be calculated using the temperature measured in the block and the derivative of the thermal impedance $\boldsymbol{Z}$ th $\boldsymbol{d}_{\boldsymbol{I}}$ determined previously. This calculated power is called $P_{\text {calc }}$ and is obtained by the following equation:

$$
P_{\text {calc }}=\operatorname{inv}\left(\text { Zthd }_{\mathbf{1}}\right) \cdot \theta_{1} / \delta \tau
$$

Knowing the power losses $P_{\text {calc }}$ allows calculating the junction temperature $T_{j \text {-calc }}$.

$$
T_{j-\text { calc }}=\boldsymbol{Z t h d}_{\mathbf{j}} \cdot P_{\text {calc }} . \delta \tau+T_{1}(0)
$$

Finally, the evolution of power losses as a function of the junction temperature can be determined.

In order to evaluate the proposed method, thermal simulations are performed using Flotherm software, which allows calculating the temperature distribution in the component-block system based on finite volume method (FVM). These data are exported in Matlab software for processing and the proposed method is implemented to simultaneously estimate the losses and the junction temperature of the tested device.

\section{Simulation OF THE MeTHOD}

To clarify the proposed method, the relationship between the total losses (including the switching losses and the conduction losses) and the junction temperature $P\left(T_{j}\right)_{\text {ref }}$ of a commercial component is firstly established theoretically according to its datasheet. The studied Si MOSFET (IRF640N) presents a significant variation in the conduction losses versus temperature. However, the losses calculation from the datasheet is not depicted because it is out of the scope of this paper [17].

Then, the losses depending on the junction temperature are applied in the thermal model shown in Fig. 1. The temperature within the block as well as the junction temperature of the device are calculated in the time domain with a time step of $0.3 \mathrm{~s}$. This time step value is chosen to limit the calculation time while obtaining relatively accurate results.

In order to limit the temperature increase, copper $(\mathrm{Cu})$ is chosen as the block material because of its good thermal conductivity $\left(\lambda_{C u}=385 \mathrm{~W} \cdot \mathrm{m}^{-1} \cdot \mathrm{K}^{-1}\right.$ at $\left.0^{\circ} \mathrm{C}\right)$. Besides, the variation of its thermal conductivity with temperature is very small $\left(0.06 \mathrm{~W} \cdot \mathrm{m}^{-1} \cdot \mathrm{K}^{-1}\right)$ and allows the thermal model being almost independent of the test conditions.

The thermal model described in section II-A is simulated with a semi-conductor device mounted on a copper block of size $10 \mathrm{~cm} \times 5 \mathrm{~cm} \times 2.5 \mathrm{~cm}$ and fixed in the middle of the larger surface area. The thermal model of the MOSFET incorporates the copper baseplate, the $\mathrm{SnAgSb}$ solder and the silicon chip which is the power dissipation source.

In the first simulation with constant power, thermal impedances $Z t h_{j}, Z t h_{l}$ and their derivatives are calculated based on simulated temperatures illustrated in Fig. 2. In a second step, with a dissipated power $P_{r e f}$ varying with time, the temperature $T_{I}$ in the block and the chip temperature $T_{j-\text {-ref }}$ are calculated using Flotherm. These values and the related thermal impedances are then used to solve the inverse problem.

As presented in section II, the proposed method uses the measurement of $T_{l}$ and the calculation of $Z t h_{l}$ to deduce the losses $P_{\text {calc }}(8)$. After that, based on this value and the derivative of $Z t h h_{j}$, the junction temperature $T_{j \text {-calc }}$ is determined as a function of time (9). Besides comparing these values with the reference losses $P_{\text {ref }}$ and the reference temperature $T_{j \text {-ref }}$ calculated by Flotherm (Fig. 3), the evolution of losses as a function of junction temperature $P\left(T_{j}\right)_{\text {calc }}$ is also deduced and compared with the reference $P\left(T_{j}\right)_{\text {ref }}$ (Fig. 4).

Fig. 3 shows the similarity between the calculated losses, the estimated junction temperature and their references. Nevertheless, it should be noticed that the calculated values are different from the reference ones at the beginning (from 0 to 5 seconds) as shown in the small figure on the lower right of Fig. 3. 


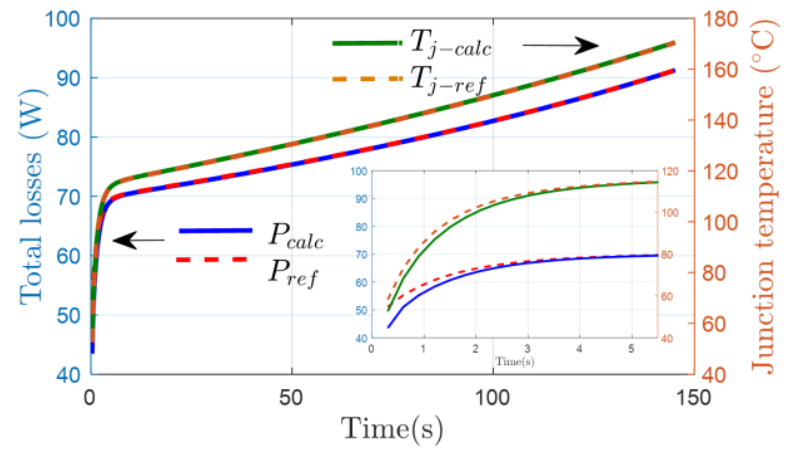

Fig. 3. Comparison between the calculated total losses, the estimated junction temperature and their references in the time domain (sample time $=0.3 \mathrm{~s}$ )

Fig. 4 depicts the curve of the imposed losses as a function of the reference junction temperature and the curve of the estimated losses as a function of the estimated junction temperature. Simulation results show that the losses are estimated with high accuracy (less than $1 \%$ of errors) at high temperatures (reached after $5 \mathrm{~s}$ ). Nevertheless, for low temperatures, there is a non-negligible error in the calculated results, which can be explained by the rapid increase of temperatures at the first moment and which depends on the time step (4). Indeed, the sample time $(0.3 \mathrm{~s})$ is quite significant compared to the temperature dynamics, especially at the very beginning of the test when the temperature increases rapidly. The obtained results can be improved by increasing the sampling frequency. The effect of sampling frequency on the accuracy on the results will be detailed in section IV-C.

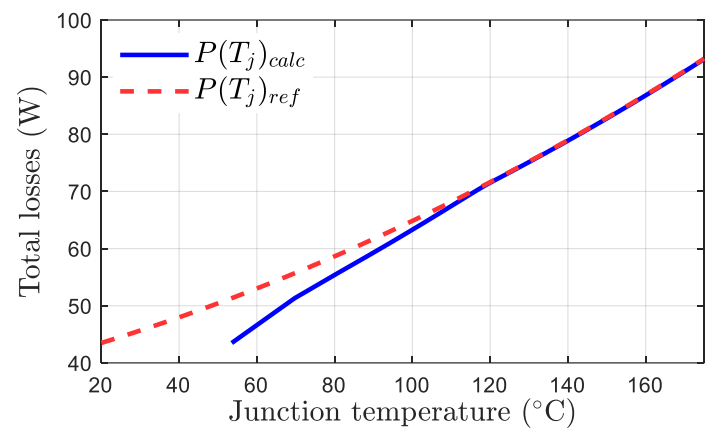

Fig. 4. Imposed and estimated dissipated powers as a function of temperature $($ sample time $=0.3 \mathrm{~s})$

Despite the satisfactory results provided by these simulation results, many practical parameters can introduce errors in the losses estimation using the proposed method. Some physical parameters of the system (block material properties, boundary conditions, thermal contact resistance ...) have a huge influence on the results. That is why the design of the system (block dimensions and materials, thermal insulation, thermal interface material...) must be carried out with care. Furthermore, the measurement error, the measurement noise, also the sampling frequency have considerable effects on the results.

The influence of these different parameters will be discussed below.

\section{ROBUSTNESS OF THE METHOD}

\section{A. Thermal contact resistance}

The thermal interface resistance value between the component and the block has a great importance. As can be seen in Fig. 5, the temperature jump of $T_{j}$ that follows the power injection increases with the thermal contact resistance. In this figure, the value of $10^{-5} \mathrm{~K} \cdot \mathrm{m}^{2} \cdot \mathrm{W}^{-1}$ corresponds to the thermal resistance of a good thermal grease which is not electrically insulating. The other values are related to classical isolating thermal interface materials like elastomers. Thus, the choice of the thermal interface material is decisive to extend the measurement range and a thermal interface material with the lowest thermal resistance must be chosen.

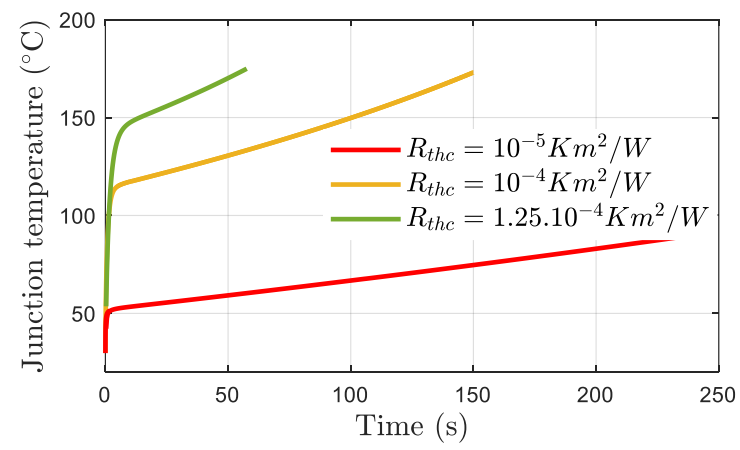

Fig. 5. Junction temperature as a function of time with different thermal contact resistances

\section{B. Boundary conditions}

This section aims at studying the influence of boundary conditions on the performance of the proposed method in order to determine whether it is necessary to thermally isolate the system. With a non-isolated block, the conditions of thermal exchange may vary from one test to another. Besides, the heat transfer coefficient, which depends on the temperature, can also evolve.

In these simulations, the different faces of the system are assumed to be no longer isolated: the convection heat transfer coefficient can be changed in different tests. For example, a convective heat transfer coefficient of $5 \mathrm{~W} \cdot \mathrm{m}^{-2} \cdot \mathrm{K}^{-1}$ is assigned during the thermal model identification step and a value of 15 $\mathrm{W} \cdot \mathrm{m}^{-2} \cdot \mathrm{K}^{-1}$ is imposed during the losses measurement step.

Fig. 6 presents the results of the loss estimation obtained with the proposed method using the thermal model determined in the first step, $P_{\text {calc }}$, and the reference losses, $P_{\text {ref }}$, which is the imposed power in the step of losses measurement. As can be noticed, the losses estimation moves away from the reference value with a difference of $2 \%$ after $140 \mathrm{~s}$. Thus, the experimental implementation of this method requires a reasonable control of the boundary conditions, e.g. insulating layers of very good performances must be fixed around the block. 


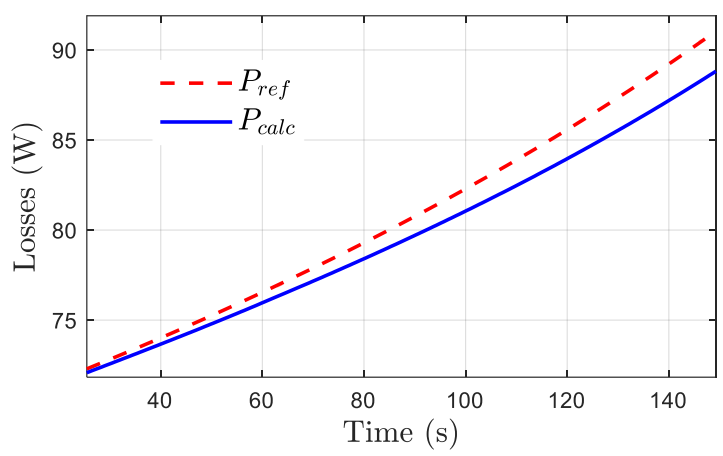

Fig. 6. Estimated and reference losses as a function of time applying different heat transfer coefficients at the block walls $\left(5 \mathrm{~W} \cdot \mathrm{m}^{-2} \cdot \mathrm{K}^{-1}\right.$ during the characterization step, and $15 \mathrm{~W} \cdot \mathrm{m}^{-2} \cdot \mathrm{K}^{-1}$ during the loss measurement step)

\section{Sampling frequency}

Increasing the sampling frequency brings benefits to the proposed method. In an ideal case, by increasing the sampling frequency, the number of measured data is greater and the accuracy of the measurements is improved, especially at the first moment during the fast rise of the junction temperature. Fig. 7 shows the evolution of the calculated losses as a function of the junction temperature recovered by the proposed method for two different time steps. The results obtained with a time step of $0.01 \mathrm{~s}$ allow estimating the losses over a wider temperature range than those obtained with a greater time step of $0.3 \mathrm{~s}$.

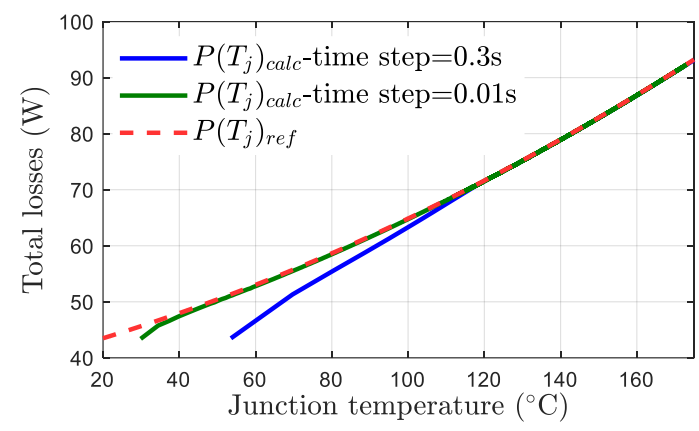

Fig. 7. Evolution of the losses as a function of the junction temperature calculated in an ideal case, with two different time steps

Therefore, to obtain accurate results on a large temperature range, it is necessary to use a high speed acquisition chain. The necessary sampling frequency depends on the speed of the temperature rise in the very first moment. For example, with a thermal contact resistance $R_{\text {thc }}=10^{-4} \mathrm{~K} \cdot \mathrm{m}^{2} \cdot \mathrm{W}^{-1}$, the chip temperature increases from 23 to $35^{\circ} \mathrm{C}$ (an increase of $12^{\circ} \mathrm{C}$ ) in the first 0.01 seconds. So, in order not to exceed $1^{\circ} \mathrm{C}$ between each sample, the data acquisition system should have, a sampling rate higher than 1200 samples per second.

\section{Measurement errors}

Systematic and repeated errors have less influence on the results because they will be identical during thermal model identification and loss measurement steps. For example, in the case where the measured temperature value always has the same offset error due to a problem that persists throughout the entire experiment, (3) shows that it will not cause any issue on the result.

On the contrary, it seems that the errors brought by the instrumentation, which are different during various tests, have a considerable effect on the results, especially at the very beginning of the experiment when the temperature is low. For example, for an error of $1^{\circ} \mathrm{C}$ in the measurement of the block temperature, the error of the estimated losses is about $3 \%$ in the first 10 seconds and decreases over time. Consequently, the determination of junction temperature is flawed, the temperature estimation error is about $10^{\circ} \mathrm{C}$ in the first 10 seconds (Fig. 8).

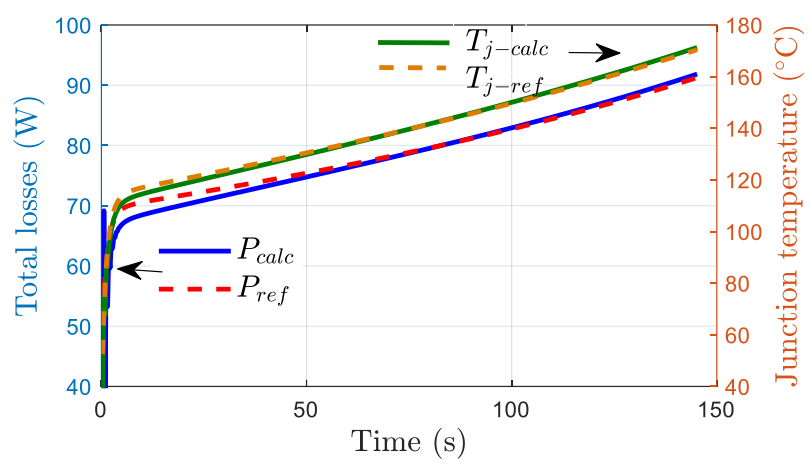

Fig. 8. Calculated total losses and estimated junction temperature in the time domain due to measurement error

It is therefore necessary to use an instrumentation chain as accurate as possible and as independent as possible from external conditions.

\section{E. Measurement noise}

In practice, temperature measurements are noisy. Thus, to consider this phenomenon, noise is artificially added to the simulated temperatures. A normal distribution function $\mathrm{N}(0$, $\sigma^{2}$ ) (e.g. $\sigma=0.1$, equivalent to a noise of $\pm 0.3^{\circ} \mathrm{C}$ ) is used to artificially produce measurement noise.

Firstly, the influence of the measurement noise can be reduced by filtering data of the acquired temperatures: a moving average filter of length $\mathrm{N}$ takes the average of every $\mathrm{N}$ consecutive samples of the waveform (e.g. to average the samples in 3 seconds, $\mathrm{N}=10$ for a time step of $0.3 \mathrm{~s}$, or $\mathrm{N}=300$ for a time step of $0.01 \mathrm{~s}$ ).

However, the results can also be divergent. This phenomenon can be explained by the calculation of the inverse of the thermal impedance matrix inv(Zthd) (8) which is carried out by the direct method (function "inv" in Matlab).

$$
\begin{aligned}
\boldsymbol{i n v}(\text { Zthd })= & \frac{1}{|\boldsymbol{Z t h d}|} \operatorname{adj}(\text { Zthd }) \\
& =\frac{1}{Z \dot{t h}(0)^{N}} \operatorname{adj}(\text { Zthd })
\end{aligned}
$$

Indeed, the smaller the $Z \dot{t h}(0)$ on the diagonal of the matrix, 
the larger the $\boldsymbol{i n v}(\boldsymbol{Z t h d})$. To solve this problem, it is possible to add artificially a small value $\varepsilon$ to the diagonal element of the matrix Zthd [18]. However, this value should be as low as possible in order to not lead to significant errors in the results. A compromise is therefore to be found, and the small value $\varepsilon=$ 0.001 is chosen here to ensure the convergence.

$$
\text { Zthd }=\text { Zthd }+\varepsilon . \mathrm{I}
$$

This addition is not always necessary but it can solve the problem of divergence in some cases and improve the results for other cases.

\section{EXPERIMENTAL VALIDATION}

\section{A. Experimental setup}

To verify the proposed method, an experimental setup is implemented with a power MOSFET (IRF640N) fixed by screw onto a copper block having the same dimensions as those of the model. All the outer faces of the system are isolated with insulating layers $\left(0.045 \mathrm{~W} \cdot \mathrm{m}^{-1} \cdot \mathrm{K}^{-1}\right.$ of thermal conductivity, 4.4 $\mathrm{cm}$ of thickness) (Fig. 9). The thermal grease (S606C, $t$-Global Technology) with thermal conductivity of $5 \mathrm{~W} \cdot \mathrm{m}^{-1} \cdot \mathrm{K}^{-1}$, is inserted between the component and the block to minimize the thermal contact resistance. In all cases, the pre-characterization and measurements must be done under the same conditions.

The temperature $T_{l}$ in the block is measured by a Pt100 probe (TC Direct) with $1 \mathrm{~mm}$ of diameter placed in a $8 \mathrm{~mm}$ depth hole inside the block - its position is shown in Fig. 1.

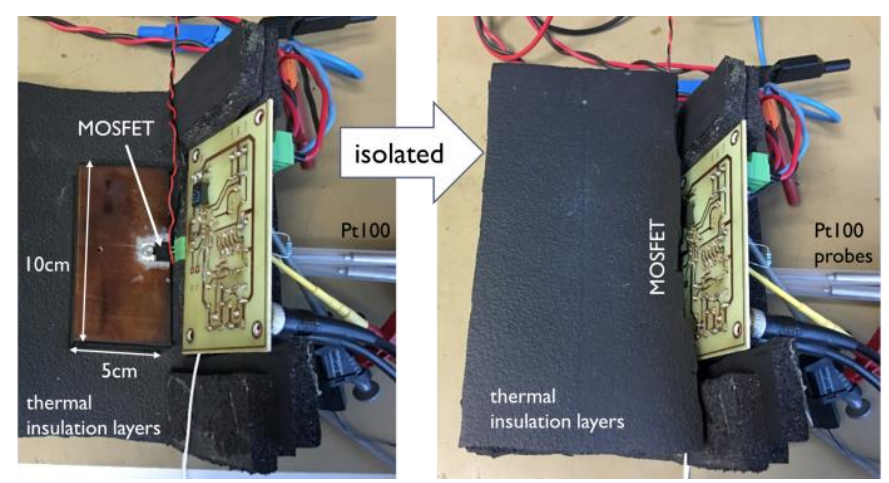

Fig. 9. Test bench

In order to avoid self-heating of the Pt100 probe and ensure the precision of temperature measurement, a low current of 0.1 $\mathrm{mA}$ is imposed using a current source (Series 2400 Source Meter Unit Instruments, Keithley) with an accuracy of \pm $(0.031 \%+20 \mathrm{nA})$. An instrumentation amplifier (ISL28534, Intersil) with a gain of 100 is added in order to amplify the voltage coming from the temperature sensor, because of its good characteristics: low input offset voltage $(0.62 \mu \mathrm{V})$, low gain error $( \pm 0.3 \%)$ and high bandwidth $(23 \mathrm{kHz})$. All measured values are processed by a portable data recorder (GEN 3i, GN610 isolated input card, $H B M$ ) with a sampling frequency of $10 \mathrm{kHz}$ and an accuracy of $0.075 \%+400 \mu \mathrm{V}$ (full scale).

A pre-calibration of the Pt100 sensor is implemented in order to increase accuracy of the measurements. To recover an accurate relation between the temperature and the measured voltage from the sensor, the calibration uses a precision probe Pt100 (TC Direct). This high precision probe presents an accuracy of $\pm 0.06^{\circ} \mathrm{C}$ at $0^{\circ} \mathrm{C}$. It is connected to a digital multimeter $(34461 \mathrm{~A}$, Keysight $)$ with an accuracy of $\pm 0.05^{\circ} \mathrm{C}$.

\section{B. Methodology for experimental tests}

The experimental verification of the proposed method is done in three steps. First, a thermo-sensitive electrical parameter (TSEP) is calibrated as a function of the junction temperature of the component. This parameter will be used later to find the thermal impedance $Z t h_{j}$, also to estimate the chip temperature which is compared with the estimated temperature obtained by the proposed method. Then, the thermal model of the system is identified. Finally, the proposed method is applied when the component is kept in conduction state and the imposed power evolves over time. Based on the given constant drain-source voltage and the controlled current through the component, the electrical power is easy to measure and can be compared with the results of the proposed calorimetric method.

\section{Calibration of the thermo-sensitive electrical parameter}

There are several thermo-sensitive electrical parameters that can be used for chip temperature estimation. For reasons of simplicity of implementation, the gate-source voltage $V_{g s, I d}$ in a linear mode is chosen as TSEP [19].

The electrical circuit shown in Fig. 10 is used for calibration. It allows measuring the variation of the gate-source voltage $V_{g s}$ with temperature under constant drain current $I_{d}$ and constant drain-source voltage $V_{d s}(15 \mathrm{~V})$. The regulated current $I_{d}$ flowing through the component is sequentially implemented with several values from $0.76 \mathrm{~A}$ to $5.15 \mathrm{~A}$ by changing the value of the input voltage $V_{i n}$.

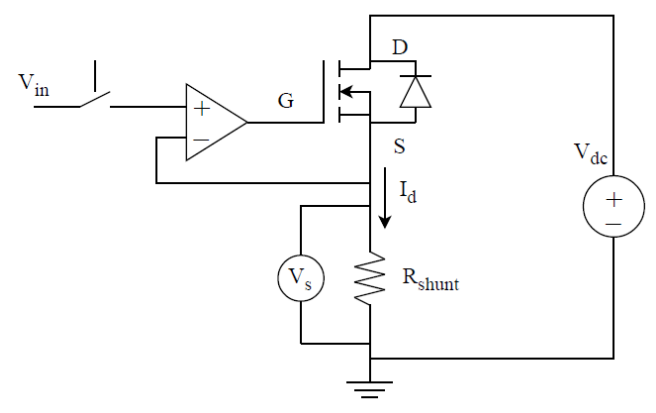

Fig. 10. Electrical circuit for experimental tests

The temperature is controlled by a hotplate (PC-600D, Corning) on which the copper block is mounted. The system is isolated and the measurement is made at least 30 minutes after the temperature setting to allow the system to reach its thermal equilibrium.

TSEP $V_{g s, I d}$ is measured for each temperature $T_{j}$ and current value $I_{d}$. But, as the dissipated power in the chip $\left(V_{d c} . I_{d}\right)$ induces an increase of the chip temperature and results in the decrease of $V_{g s}$, short current pulses ( $2 \mathrm{~ms}$ ) are generated in order to limit 
the self-heating of the device.

The value of $V_{g s, I d}$ is determined by calculating the initial value $V_{g s}(0)(t=0$ corresponds to the first moment when the component is turned on). To measure this value, a linear extrapolation as a function of the square root of time $V_{g s}(\sqrt{t})$ can be established [19]. Ten measurements are taken to average out this value.

Then, by varying the temperature, the gate-source voltage is deduced as a function of the junction temperature at several current levels (Fig. 11).

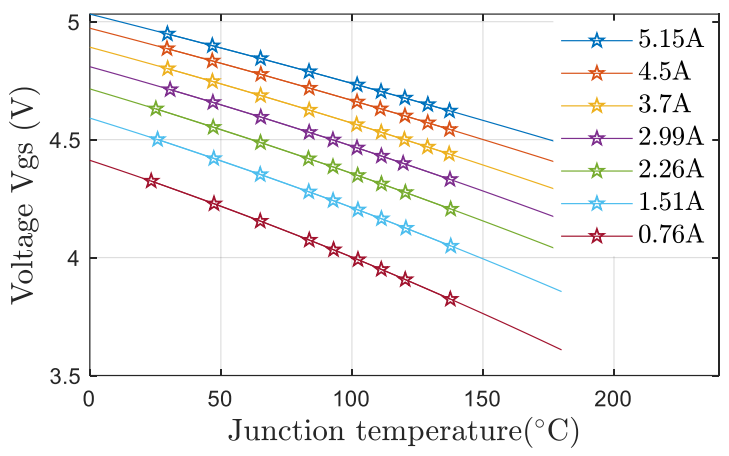

Fig. 11. Gate-source voltage as a function of the junction temperature $V_{g s, I d}\left(T_{j}\right)$ at different current levels

The sensitivity of this thermo-sensitive electrical parameter depends on the semi-conductor chip. It is in the $-4.3 \mathrm{mV} /{ }^{\circ} \mathrm{C}$ (for $0.76 \mathrm{~A}$ ) to $-3 \mathrm{mV} /{ }^{\circ} \mathrm{C}$ (for $5.15 \mathrm{~A}$ ) range for the chosen device. The set of results was fitted by an interpolation function allowing, for each value of the current $I_{d}$ and each value of the voltage $V_{g s}$, to estimate the junction temperature.

\section{Thermal model identification}

To identify the thermal model, we set up the component to operate at constant power for a long period of time (300 s). A known power $P$ is injected into the system by fixing the voltage $V_{d s}(15 \mathrm{~V})$ and the current $I_{d}(4.2 \mathrm{~A})$ flowing through the component.

As mentioned in section IV.E, a moving average filter of length $\mathrm{N}$ is used after measuring temperature $T_{l}$ and estimating junction temperature $T_{j}$ (Fig. 12) to reduce the influence of the measurement noise. Moreover, due to the memory limit in Matlab software, the number of samples should be reduced to be able to resolve the equation (8) and (9) (time step of $10 \mathrm{~ms}$ instead of $100 \mu \mathrm{s})$.

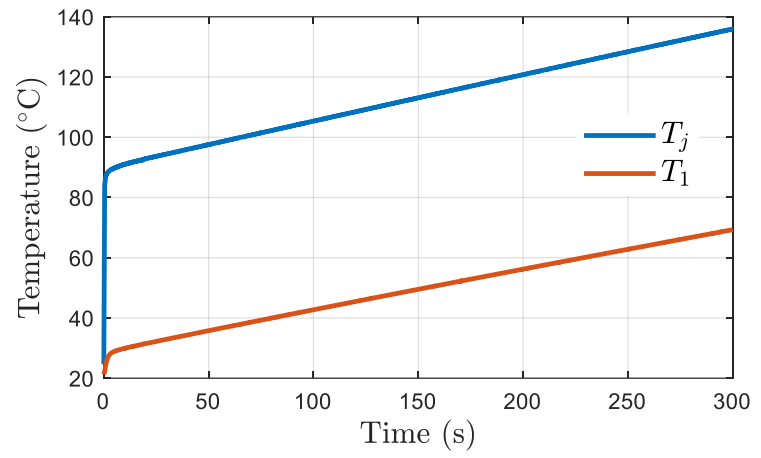

Fig. 12. Measured temperature $T_{l}$ and estimated junction temperature $T_{j}$ as a function of time for $P=62.8 \mathrm{~W}$ (time step of $10 \mathrm{~ms}$ )

The thermal impedance from the temperature measuring point to the ambient, $Z t h_{l}$, and the thermal impedance from the component junction to the ambient, Zth $h_{\text {, are calculated }}$ respectively based on the temperature $T_{l}$ measured in the block, and the junction temperature $T_{j}$ estimated with the thermosensitive electrical parameter. As can be noticed, the derivatives of these thermal impedances increase quickly in very first moments, after that, their values decrease and are almost constant over longer times $\left(2.5 \cdot 10^{-3} \mathrm{~K} \cdot \mathrm{W}^{-1} \cdot \mathrm{s}^{-1}\right.$ for $Z \dot{t} h_{\mathrm{j}}$ and $2.25 \cdot 10^{-3} \mathrm{~K} \cdot \mathrm{W}^{-1} \cdot \mathrm{s}^{-1}$ for $Z \dot{t} h_{1}$ ) (Fig. 13).
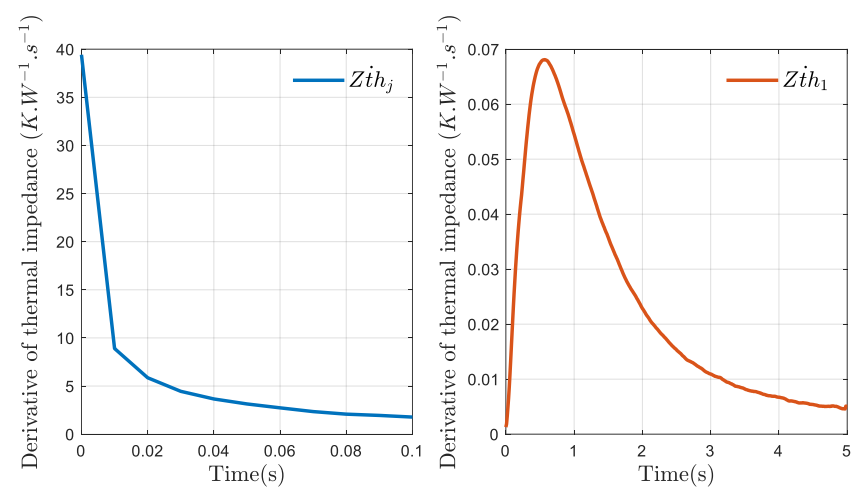

Fig. 13. Derivative of thermal impedance $Z \dot{t} h_{j} \& Z \dot{t} h_{l}$ (time step of $10 \mathrm{~ms}$ )

\section{E. Method validation with variable power}

To validate the proposed method, a dissipated power varying with time is injected into the transistor. The same circuit shown in Fig. 10 is applied with a fixed-voltage $V_{d c}$ and the current $I_{d}$ which varies as a function of time (ramp function - Fig. 14).

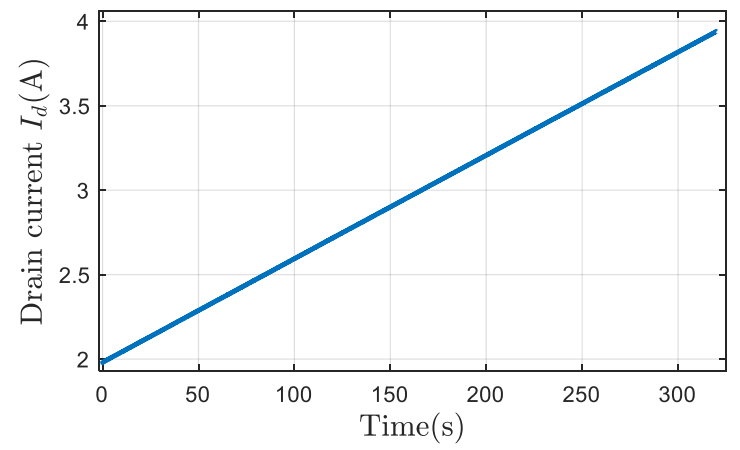

Fig. 14. Drain current variation with time (time step of $10 \mathrm{~ms}$ ) 
The temperature $T_{1}$ in the block is measured to deduce the power $P_{\text {calc }}(t)$, then $V_{d s}(t)$ and $I_{d}(t)$ are acquired to calculate $P_{\text {ref }}(t)$. The comparison between both quantities in Fig. 15 shows a good agreement. An estimate of the difference between the two quantities is calculated using (12) and is estimated to be less than $2 \%$ at the beginning and less than $0.5 \%$ after a few seconds.

$$
\text { Uncertainty }=\frac{P_{\text {ref }}-P_{\text {calc }}}{P_{\text {ref }}} .100 \%
$$

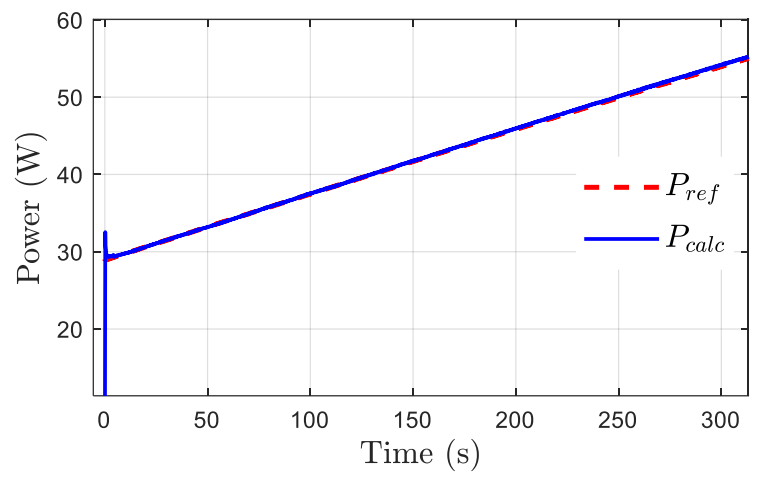

Fig. 15. Reference and estimated dissipated power as a function of time

Then, the junction temperature $T_{j \text {-calc }}$ is estimated as a function of time (Fig. 16) using $P_{\text {calc }}$ and the derivative of the thermal impedance $Z \dot{t} h_{\mathrm{j}}$. It is also possible to estimate the junction temperature $T_{j-e s t}$ through TSEP $V_{g s}(t)$ and the measured current $I_{d}(t)$. Another value, $T_{j-r e f}$, is deduced by using the value of $P_{\text {ref }}$ and $Z t h_{\mathrm{j}}$.

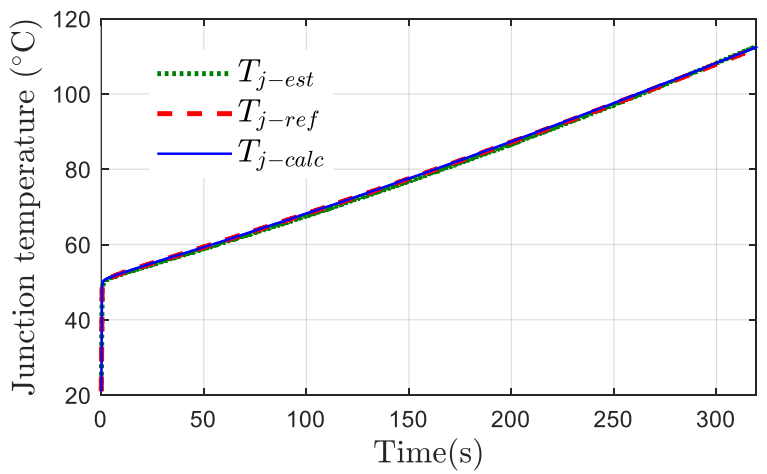

Fig. 16. Calculated and estimated junction temperature as a function of time

The experimental results are acceptable after $1 \mathrm{~s}$, with an error which is lower than $\pm 2{ }^{\circ} \mathrm{C}$.

Finally, by measuring the increase of local temperature in a copper block fixed under the component, in conduction state, the proposed method allows recovering the dissipated power and estimating the junction temperature of the device based on this calculated power.

\section{F. Analysis of estimation errors}

The loss and junction temperature estimation errors are due to two processes that are necessary for the implementation of the method: electronic measurements (acquisition chain) and mathematical treatments.

About the acquisition chain, the accuracies of the measurement tools are given in section V.A. It is thus possible to estimate the error during the loss measurement $\left(V_{d s} . I_{d}\right)$. The error on $V_{d s}$ is due to the data recorder and is estimated to be $0.1 \%$. The error on $I_{d}=V_{s} / R_{\text {shunt }}$ is due to the recorder $(0.2 \%)$ and to $R_{\text {shunt }}$ variation with temperature. Knowing the temperature coefficient of $R_{\text {shunt }}\left(100 \mathrm{ppm} /{ }^{\circ} \mathrm{C}\right)$ and estimating that the temperature increase of this device is lower than $50^{\circ} \mathrm{C}$, it is estimated that the global error on $\mathrm{I}_{\mathrm{D}}$ is less than $0.7 \%$. Therefore, the error concerning the power estimation is $0.8 \%$. Note that the main contribution of this error is due to the shunt resistor and could be easily reduced.

The temperature measurement error is due to the probe and multi-meter defects (error less than $0.11^{\circ} \mathrm{C}$ ), the instrumentation amplifier and the data recorder accuracies. It is estimated to be $1^{\circ} \mathrm{C}$, the main error being due to the gain of the amplifier.

Since the temperature measurement is made with the same acquisition chain, the error on $\theta_{l}$ and $\theta_{j}(3)$ should be cancelled. However, in the worst case, it could be $2^{\circ} \mathrm{C}$. Therefore, the maximum error decreases from $10 \%$ to $2 \%$, corresponding to the temperature variation between $20^{\circ} \mathrm{C}-100^{\circ} \mathrm{C}$. The thermal impedance measurement ((6), (7)) has the total error of temperature variation and that of the power estimation in the range of $0.8 \%-10.8 \%$.

The actual losses can be estimated (8) with the error of approximately $0.8-11.6 \%$ and the junction temperature is deducted from (9) with the error between $2^{\circ} \mathrm{C}-10^{\circ} \mathrm{C}$. The considerable error of junction temperature is also caused by the variation of shunt resistance value. So, choosing a fixed value of this resistance and using the same acquisition chain during experimental tests can reduce these errors.

About the mathematical treatment, the errors are linked to the chosen sampling frequency (4) and the value of $\varepsilon$ (11). A simulation performed with a sampling period of $10 \mathrm{~ms}$ and $\varepsilon=$ 0.001 gives the estimation error of $0.2 \%$ for the calculation of loss and $0.2^{\circ} \mathrm{C}$ for the junction temperature evaluation (after $1 \mathrm{~s})$.

The global estimated losses and temperature are compared with the reference value, however, which also has the error in practical measurement. Therefore, the difference between these parameters is approximately $2 \%$ for the power and $3{ }^{\circ} \mathrm{C}$ for junction temperature. It confirms the practical errors obtained during the experimental work.

To complete the analysis, it must be noted that, when using this method in power converters, the high switching speed of the component (the value of $\mathrm{dV} / \mathrm{dt}$ ), and the capacitive couplings between heat sink and power switches can induce interferences that disturb the temperature measurements. These problems must be solved to ensure the feasibility of this method. Furthermore, some signal processing techniques after collecting data can be used to reduce the noise.

\section{Conclusion And Perspectives}

A new method for estimating losses based on the notion of 
thermal impedance has been proposed using transient temperature measurements. The proposed method inherits and improves the other calorimetric methods: the measurement time is shorter than that of steady state conventional calorimetric methods and the implementation is carried out under simple measurement conditions. Moreover, this method can evaluate the junction temperature of the component at which the losses are estimated, while the junction temperature is generally controlled poorly in other calorimetric methods. In addition to the evaluation of this method, thermal simulations with Flotherm software have made it possible to analyse the influence of the system's physical parameters and measurement errors/noises. It is important to minimize contact resistance, thermally insulate the experimental system, use a highprecision instrumentation chain and an appropriate sampling frequency to obtain accurate results. In addition, signal postprocessing also helps to reduce the influence of measurement noise. By applying these conditions, an experimental approach was then implemented to verify this method. The results obtained are extremely promising with an error in the estimation of power below $2 \%$ and that in the determination of the junction temperature below $2{ }^{\circ} \mathrm{C}$. The feasibility of the proposed method is therefore confirmed. Further work will be devoted to validate the proposed method with components used in a switching cell and compare these results with other methods. The challenge now is the limitation of the interferences in high switching converters that can affect the noise and accuracy of measurements. Then, this method will be evaluated for the measurement of losses in several components fixed on the same block and/ or in multi-chip modules.

\section{REFERENCES}

[1] Z. Zhang, B. Guo, F. Wang, L. M. Tolbert, B. J. Blalock, Z. Liang, and P. Ning, "Methodology for switching characterization evaluation of wide band-gap devices in a phase-leg configuration," in Applied Power Electronics Conference and Exposition (APEC), 2014 Twenty-Ninth Annual IEEE. IEEE, 2014, pp. 2534-2541.

[2] D. Oustad, S. Lefebvre, M. Petit, D. Lhotellier, and M. Ameziani, "Comparison of modeling switching losses of an igbt based on the datasheet and an experimentation," in Power Electronics and Applications (EPE'16 ECCE Europe), 2016 18th European Conference on IEEE, 2016, pp. 1-10.

[3] F. Forest, J.-J. Huselstein, S. Faucher, M. Elghazouani, P. Ladoux, T. Meynard, F. Richardeau, and C. Turpin, "Use of opposition method in the test of high-power electronic converters," IEEE Transactions on Industrial Electronics, vol. 53, no. 2, pp. 530-541, 2006.

[4] J. Brandelero, B. Cougo, T. Meynard, and N. Videau, "A non-intrusive method for measuring switching losses of gan power transistors," in Industrial Electronics Society, IECON 2013-39th Annual Conference of the IEEE. IEEE, 2013, pp. 246-251.
[5] K. Zou and C. Chen, "A power loss characterization method for semiconductor switching devices based on inverter-level dc measurements," in Applied Power Electronics Conference and Exposition (APEC), 2014 Twenty-Ninth Annual IEEE. IEEE, 2014, pp. 1287-1292.

[6] W. Cao, K. J. Bradley, and A. Ferrah, "Development of a high-precision calorimeter for measuring power loss in electrical machines," IEEE Transactions on Instrumentation and Measurement, vol. 58, no. 3, pp. 570-577, 2009.

[7] D. Christen, U. Badstuebner, J. Biela, and J. W. Kolar, "Calorimetric power loss measurement for highly efficient converters," in Power Electronics Conference (IPEC), 2010 International. IEEE, 2010, pp.1438-1445.

[8] A.-S. Podlejski, C. Buttay, D. Bergogne, J. Morand, C. Vollaire, and A. Bréard, "Mesure précise des pertes dans un module d'électronique de puissance par une méthode calorimétrique," in Symposium de Génie Electrique 2014, 2014.

[9] P. Grzejszczak and R. Barlik, "Switching losses in a new high-voltage mosfets," in Progress in Applied Electrical Engineering (PAEE). IEEE, 2016, pp. 1-6.

[10] D. Iero, F. G. Della Corte, G. Fiorentino, and P. M. Sarro, "A calorimetry-based measurement apparatus for switching losses in high power electronic devices," in Energy Conference (ENERGYCON), 2016 IEEE International. IEEE, 2016, pp. 1-5.

[11] L. Hoffmann, C. Gautier, S. Lefebvre, and F. Costa, "Optimization of the driver of gan power transistors through measurement of their thermal behavior," IEEE Transactions on Power Electronics, vol. 29, no. 5, pp. 2359-2366, 2014.

[12] C. Chen, D. Labrousse, S. Lefebvre, H. Morel, C. Buttay, J. Andr'e, and M. Domeij, "Power loss estimation in sic power bjts," in PCIM Europe 2014; International Exhibition and Conference for Power Electronics, Intelligent Motion, Renewable Energy and Energy Management; Proceedings of VDE, 2014, pp. 1-8.

[13] D. Rothmund, D. Bortis, and J. W. Kolar, "Accurate transient calorimetric measurement of soft-switching losses of 10-kv sic mosfets and diodes," IEEE Transactions on Power Electronics, vol. 33, no. 6, pp. 5240-5250, 2018.

[14] A. Anurag, S. Acharya, Y. Prabowo, G. Gohil, H. Kassa, and S. Bhattacharya, "An accurate calorimetrie method for measurement of switching losses in silicon carbide (sic) mosfets," in Applied Power Electronics Conference and Exposition (APEC), 2018 IEEE. IEEE, 2018, pp. 1695-1700.

[15] Y. Avenas, L. Dupont, and Z. Khatir, "Temperature measurement of power semiconductor devices by thermo-sensitive electrical parameters - a review," IEEE Transactions on Power Electronics, vol. 27, no. 6, pp. 3081-3092, 2012.

[16] Y. Gerstenmaier and G. Wachutka, "A new procedure for the calculation of the temperature development in electronic systems," in EPE99 conference, 1999.

[17] J. Brown, "Power mosfet basics: Understanding gate charge and using it to assess switching performance," Vishay Siliconix, AN608, 2004.

[18] J.-P. Barbot, "Identication et problemes inverses : diverses approches possibles."

[19] Y. Avenas and L. Dupont, "Evaluation of igbt thermo-sensitive electrical parameters under different dissipation conditions-comparison with infrared measurements," Microelectronics Reliability, vol. 52, no. 11, pp. 2617-2626, 2012. 